\title{
QUESTÕES SOBRE A INTEGRAÇÃO DE NOVOS MEIOS DE MOBILIDADE AO PLANO DE MOBILIDADE URBANA E TRANSPORTE DE CURITIBA
}

\author{
ISSUES REGARDING THE INTEGRATION OF NEW MEANS OF \\ MOBILITY INTO CURITIBA'S URBAN MOBILITY AND TRANSPORT \\ PLAN
}

\begin{abstract}
Andrea Martinesco Coelho Martins ${ }^{1}$
\section{RESUMO}

A análise do Plano de Mobilidade Urbana e Transporte Integrado da Prefeitura Municipal de Curitiba demonstra a grande preocupação dos agentes públicos com o desenvolvimento de Políticas Públicas no campo da mobilidade urbana, envolvendo, para o que interessa no presente trabalho, o transporte coletivo e o transporte comercial metropolitano. Nesse sentido, foi realizado estudo sobre os novos usos relacionados aos serviços de mobilidade compartilhada desenvolvidos na Europa, em especial na França, para identificação de alternativas viáveis aos problemas de saturação do sistema viário nas áreas urbanas e suburbanas de Curitiba, visando ao transporte urbano sustentável. Serão apresentados dois sistemas alternativos aos descritos no PlanMob Curitiba - covoiturage e autopartage -, introduzidos na Europa desde a década de 1980. Serão estudadas as características de cada sistema e avaliadas as estatísticas francesas. O resultado da pesquisa indicou que, naquele país, pela crescente demanda por alternativas ao uso do petróleo, associada às mudanças climáticas, bem como à saturação das redes de mobilidade, há um esforço multidisciplinar na formação e adoção de novos sistemas de mobilidade eco-sustentáveis, que passa pelo desenvolvimento do veículo elétrico, também chamado de carro verde (com emissão "zero" de carbono), a delegação da condução e a conectividade, que vão além do proposto nesse trabalho.
\end{abstract}

Palavras-chave: Transporte urbano sustentável; Covoiturage; Autopartage.

\begin{abstract}
This analysis of the Local Government of Curitiba's Urban Mobility and Integrated Transport Plan shows the great concern of public agents over the development of public policies in the field of urban mobility. This issue is of interest to this study because it involves collective transport and metropolitan commercial transport. With this in mind, the study was conducted concerning the new uses regarding the shared mobility services developed in Europe, especially France, in order to identify the viable alternatives to the problems of saturation of the road system in urban and suburban areas of Curitiba, with a view to developing sustainable urban transport. Two alternatives to the systems described in the PlanMob Curitiba will be presented: covoiturage and autopartage, which were introduced in Europe in the 1980s. The features and characteristics of each system will be studies and the French statistics will be analyzed. The results of the study suggested that in France, due to the increasing demands for alternatives to oil, combined with climate changes and the saturation of the mobility networks, there is a multidisciplinary effort to create and adopt new eco-sustainable mobility systems, ranging from the development of an electric vehicle, also known as a green car (with zero carbon emission) to the delegation of conduction and connectivity which lie outside the scope of this study.
\end{abstract}

Key words: Sustainable urban transport; Covoiturage; Autopartage.

Recebido 09/05/2013; Aceito 30/06/2013

\footnotetext{
${ }^{1}$ Bacharel em Direito. Especialista em Direito Tributário. É servidora do Ministério Público Federal desde 1999 e presta assessoria jurídica em processos judiciais, administrativos e extrajudiciais na área criminal, desde 2005, e na área eleitoral, desde 2012. E-mail: manhedaju@ hotmail.com
} 


\section{Introdução}

A dispersão espacial entre o local de moradia e as atividades cotidianas, juntamente com o aumento da renda média da população e a sensação de "riqueza" que a propriedade do veículo transmite a seu possuidor, contribuiu, nos últimos anos, para um aumento considerável no número de automóveis no trânsito urbano de Curitiba. Isso porque o veículo particular é considerado pelo usuário um meio mais eficaz, rápido e confortável de deslocamento, representando, na verdade, maior liberdade de locomoção.

A par de se constituir em uma alternativa ao sistema público de transporte, atualmente sobrecarregado em virtude do crescimento populacional e da metropolização, políticas econômicas adotadas pelo governo federal em 2012, em especial a redução do Imposto sobre Produtos Industrializados (IPI) para veículos zero quilômetro, culminaram no aumento da motorização em Curitiba.

Segundo dados divulgados pela Federação Nacional da Distribuição de Veículos Automotores (Fenabrave), ${ }^{2}$ as vendas de carros novos, desde o início da redução do IPI para o setor, tiveram, em agosto de 2012, o melhor mês da história da indústria automobilística brasileira, com a marca de 420.101 unidades, o que representou um aumento de 15,3\% sobre o mês de julho e de 28,3\% em relação a agosto de 2011 .

Todavia, os meios de deslocamento individuais, notadamente em veículos particulares, vêm sobrecarregando os centros urbanos, o que representa um custo elevado à sociedade, não somente em termos financeiros, mas igualmente em relação ao consumo de energia, espaço e tempo. O transporte individual, na medida em que se mostra um grande problema para as autoridades públicas e aos gestores dos espaços públicos, é a razão de ser da busca por meios de transporte alternativos, coletivos, que ajudam a desafogar o trânsito, diminuem a necessidade de vagas de estacionamento e, sobretudo, colaboram com o respeito ao meio ambiente urbano.

\section{Planejamento urbano de Curitiba}

A Prefeitura Municipal de Curitiba, em trabalho conjunto com o Instituto de Pesquisa e Planejamento Urbano de Curitiba (IPPUC) e a Urbanização de Curitiba S.A. (URBS), com o apoio de diversas entidades, como o Ministério Público do Estado do Paraná, a Fundação Getulio Vargas e a Universidade Federal do Paraná, apresentou, em março de 2008, Proposta Preliminar para o Plano de Mobilidade Urbana e Transporte Integrado (PlanMob Curitiba), elaborada em consonância com as diretrizes das políticas estabelecidas na Lei Municipal $\mathrm{n}^{\circ}$ 11.266/2004, que estabelece a adequação do Plano Diretor de Curitiba ao Estatuto da Cidade. Seu objetivo principal consistia em estabelecer políticas, diretrizes e planos de ação para o calendário de 2020, relativos à mobilidade urbana, objetivando a promoção da cidadania e inclusão social, o aperfeiçoamento institucional, regulatório e da gestão, através de ações integradas de desenvolvimento urbano, de mobilidade e de proteção ao meio ambiente.

A elaboração do PlanMob Curitiba teve um enfoque metropolitano e foi organizada em quatro temas: acessibilidade, circulação e sistema viário, sistemas de transporte coletivo e comercial e sistema de transporte de cargas.

\footnotetext{
${ }^{2}$ Disponível em <http://www.fenabrave-pr.com.br>. 
As diretrizes quanto à mobilidade estão, dentre outras, estabelecidas no Artigo 15 da Lei Municipal $n^{\circ} 11.266 / 2004$, no que interessa ao presente trabalho:

Art.15. A política municipal de mobilidade urbana e transporte tem o compromisso de facilitar os deslocamentos e a circulação de pessoas e bens no Município, com as seguintes diretrizes gerais:

I - priorizar no espaço viário o transporte coletivo em relação ao transporte individual;

II - melhorar e ampliar a integração do transporte público coletivo em Curitiba e buscar a consolidação da integração metropolitana;

III - priorizar a proteção individual dos cidadãos e do meio ambiente no aperfeiçoamento da mobilidade urbana, circulação viária e dos transportes;

[...]

IV - promover a acessibilidade, facilitando o deslocamento no Município, através de uma rede integrada de vias, ciclovias e ruas exclusivas de pedestres, com segurança, autonomia e conforto, especialmente aos que têm dificuldades de locomoção;

[...]

VII - compatibilizar o planejamento e a gestão de mobilidade urbana para promover a melhoria da qualidade do meio ambiente;

[...]

IX - estimular a adoção de novas tecnologias que visem à redução de poluentes, resíduos ou suspensão e de poluição sonora, priorizando a adoção de combustíveis renováveis;

$[\ldots]$

XI - Instituir o Plano Municipal de Mobilidade e Transporte Urbano Integrado.

Parágrafo único. As diretrizes gerais da política municipal de mobilidade urbana e transporte são voltadas para o conjunto da população do Município, com diretrizes específicas para os seus principais componentes.

Nacionalmente, Curitiba é a pioneira do Programa "Cidades Inovadoras", como o "Projeto Curitiba 2030", cujos trabalhos estão focados em sete áreas prioritárias, com maior potencial de transformar a vida dos moradores de Curitiba e da região metropolitana. São eles: Governança, Cidade em rede, Cidade do conhecimento, Transporte e mobilidade, Meio ambiente e biodiversidade, Saúde e bem-estar e Coexistência em uma cidade global.

Enquanto o PlanMob reflete a ótica do administrador público para promoção da mobilidade urbana em Curitiba e suas conexões metropolitanas, direcionando e regulando investimentos futuros na infraestrutura viária geral, o segundo busca soluções inovadoras para os problemas da cidade, visando à melhoria da qualidade de vida, com a proposta de envolver as pessoas no projeto, para que desenvolvam algum tipo de ação.

Problema posto, passa-se a tratar do objetivo deste trabalho, qual seja, a apresentação de soluções ao sistema de mobilidade urbana de Curitiba, a partir de estudos e experiências desenvolvidas na França. 


\section{A experiência francesa}

Os problemas relacionados à mobilidade de pessoas nas áreas urbanas e suburbanas envolvem, tanto no Brasil quanto na França, a saturação da rede viária e do transporte público, bem como o percurso, por vezes demasiadamente longo, entre o local de moradia e o local de trabalho e das atividades cotidianas do indivíduo.

Na França, a expressão "Novos Serviços de Mobilidade" (NSM) almeja reunir sobre uma mesma governança e serviço diferentes meios de transporte. Ela se apresenta como uma utilização diferente de um modo já existente e tradicional de mobilidade, tal como carro, bicicleta e ônibus. Tais meios de deslocamento de pessoas foram repensados e melhorados graças ao desenvolvimento tecnológico e organizacional, chegando-se, atualmente, ao covoiturage, autopartage, vélo en libre-service (VLS) e taxis compartilhados, dentre outros.

A necessidade de uma evolução conjugada de todos os atores desse ecossistema, dos construtores de carros até os órgãos governamentais de decisão, levou a França a criar, em 2013, um instituto dedicado à mobilidade sem carbono, o VeDeCoM, ${ }^{3}$ que conta com 46 participantes; grandes grupos industriais, como Renault, PSA, Valeo e Safran; organismos universitários de pesquisa; organismos governamentais e atores territoriais. Isso mostra como a nova mobilidade será complexa em multiaspectos.

Esses meios de transporte possuem diferentes definições, utilizam diferentes tecnologias e são desenvolvidos de formas distintas. Por essa razão, neste trabalho serão apresentados apenas os sistemas de covoiturage e autopartage.

Registre-se que ambos os sistemas são regulamentados em legislação específica na França, assim como em outros países europeus e nos Estados Unidos.

\subsection{O sistema autopartage}

Autopartage é um sistema de compartilhamento de veículos pertencentes a um ente público ou privado, entre pessoas diversas (SCHAEFERS, 2013). Historicamente, o sistema nasceu na Suíça, em 1948, chegando à França na década de 1970. A Suíça é considerada um modelo nesse sistema, sendo o Mobility Carsharing Suisse o principal operador europeu de autopartage. O sistema está presente, ainda, no Reino Unido, Alemanha (FIRNKORN, 2012), Canadá, Estados Unidos (MARTIN; SHAHEEN, 2011) e Singapura, considerados os mais importantes.

No autopartage, uma pessoa jurídica (v.g. de direito privado, associação ou ONG) é proprietária da frota de veículos que são colocados à disposição da pessoa que deseja locar. A reserva do veículo é feita pelo telefone ou via internet, conforme a disponibilidade do modelo escolhido.

O sistema tradicional de autopartage consiste em pegar um veículo em determinado local, sem condutor, utilizá-lo no trajeto que desejar e devolvê-lo onde foi retirado. $\mathrm{Na}$ segunda forma, conhecida como mão única (one-way ou en trace direct), pode-se pegar o veículo em uma estação e devolvê-lo em outra (DI FEBBRARO; SACCO; SAEEDNIA, 2012). Este é o que mais se aproxima do sistema da bicicleta de livre serviço (vélo en libre-

\footnotetext{
${ }^{3}$ Para mais informações, ver: <http://wwwfondation-moveotec.com/fr/la-recherche-et-linstitut-vedecom.html〉. 
service), recentemente implantado em Curitiba. ${ }^{4}$ Pesquisas realizadas na França apontam que o sistema one-way é o mais utilizado pela população, tendo em conta que disponibiliza aos usuários vagas de estacionamento especiais, próximas às estações de trem e metrô.

O sistema utiliza tecnologia de informação e comunicação, necessárias, por exemplo, para permitir, por meio de um leitor de cartão que fica afixado no para-brisa, a entrada do condutor no veículo, eis que as chaves, a priori, estarão dentro do carro. Se o veículo não possuir esse sistema, as chaves são colocadas em uma caixa de segurança próxima. $\mathrm{O}$ pagamento é feito por meio de cartão de crédito, previamente cadastrado na ficha do condutor, proporcional ao tempo de uso do veículo e aos quilômetros rodados. Os gastos da utilização incluem todos os custos relativos ao combustível, seguro, impostos e o lucro da empresa locadora. Há duas formas de adesão ao sistema, a inscrição tradicional, com a cobrança de taxa anual, e a adesão à própria empresa, que pode ser, por exemplo, uma cooperativa (BIESZCZAT; SCHWIETERMAN, 2012).

O objetivo do sistema é favorecer a mobilidade urbana combinada. Em um primeiro momento, o usuário poderá escolher o meio de transporte que melhor satisfaça às suas necessidades, como, por exemplo:

- transporte público: quando houver linha de ônibus disponível próxima da origem e do destino, em horário compatível com a sua necessidade, fora do horário de pico;

- bicicleta: particular ou no sistema de livre serviço, dependendo da distância, do condicionamento físico do usuário e das condições climáticas;

- táxi ou táxi compartilhado: para deslocamentos rápidos ou urgentes, devido ao alto custo;

- carro particular: disponível 24 horas por dia, 7 dias por semana, normalmente de acesso imediato, com custos fixos dominantes (relativo ao próprio bem, seguro, combustível, etc.);

- locação de carro tradicional: normalmente utilizado nos fins de semana ou em viagens de lazer ou à trabalho; e

- autopartage: disponível mediante reserva, com escolha do modelo de acordo com a necessidade, acesso em estações predefinidas, com custos variáveis dominantes.

Outra forma de utilização do sistema é a intermodal, quando o usuário utiliza mais de um meio de transporte para deslocamentos mais longos ou mais complexos, como são normalmente os deslocamentos interbairros sem passar pelo centro da cidade. Pode-se citar como exemplo a utilização de bicicleta até a estação de trem/metrô, o deslocamento com um desses dois meios e o autopartage até o destino final.

Estudos levados a feito na Suíça concluíram que a utilização do autopartage contribuiu para a redução da motorização das famílias que aderem a esse tipo de serviço. Cada veículo, nesse sistema, substitui de 4 a 8 veículos particulares nas ruas, o que abre perspectivas de realocação de espaços outrora utilizados para estacionamento de veículos, facilitando o fluxo urbano.

A redução de veículos nas ruas diminui os problemas de congestionamento, bem como os relacionados à emissão de gases no meio ambiente. Nesse sentido, cabe destacar que algumas frotas na Europa são compostas exclusivamente por carros elétricos ou, ainda, por veículos novos que possuem sistemas mais adequados à redução da poluição nos centros urbanos (EL FASSI; AWASTHI; VIVIANI, 2012).

\footnotetext{
${ }^{4}$ Para mais informações, ver: <http://www.bicicletaria.net>. 
Diante do exposto, entende-se que o sistema de compartilhamento de veículo denominado autopartage pode ser considerado um serviço essencial para a inovação da mobilidade urbana em Curitiba. Esta proposta inclui a atribuição de vagas de estacionamento específicas (esforço geral gerado pela coletividade) e prioritárias para esse tipo de veículo.

\subsection{O sistema covoiture}

O objetivo desse antigo sistema alternativo de mobilidade (conhecido nos anos 1970 como "carona") é aumentar o número de pessoas em um veículo (particular) dentro de um trajeto. Em outras palavras, aumentar a "taxa de ocupação" de um veículo (DELOACH; TIEMANN, 2012). A definição legal na França é que o covoiturage consiste na utilização comum de um veículo por um condutor não profissional e um ou mais passageiro(s), com o objetivo de efetuar todo o trajeto comum ou apenas parte dele (CAHOUR; NGUYEN; CRÉNO, 2013). Diferentes medidas de incitabilidade à adoção desse tipo de mobilidade podem ser encontradas em diversos países. Na Alemanha, por exemplo, diferentes governos (chamados "landers") propõem um centro de gestão da oferta e da demanda passando por um sítio central gerado pelas coletividades. ${ }^{5} \mathrm{O}$ governo federal de Westphalie Rhin Nord contabilizou o custo a 80 reais/ano, por pessoa. O serviço atende 2,2 milhões de pessoas, efetuando trajeto domicílio-trabalho, e representa, a cada dia, uma oferta entre 8 e 9 mil trajetos.

Outros estados europeus, como Reino Unido, Holanda e Bélgica, propõem sistemas similares. Existe, ainda, uma redução de impostos aplicável a pessoas testando a utilização desse tipo de transporte.

Os Estados Unidos utilizam outra modalidade incitativa para o covoiturage (carsharing), que são as vias dedicadas a carros contendo mais de um passageiro, o que permite um ganho importante de tempo de trajeto nas horas de pico.

Tanto os Estados Unidos como a Europa desenvolveram áreas de estacionamento periférico específico, dedicadas ao covoiturage, monitoradas por câmeras de segurança, permitindo que diferentes utilizadores possam estacionar os seus carros para, em seguida, dividir um único veículo no trajeto conjunto até o centro da cidade (CERTU, 2007).

No covoiturage, o deslocamento é determinado pelo condutor, normalmente são utilizados carros de pequeno porte e a atividade não visa ao lucro, mas sim à diminuição das despesas fixas do veículo particular, bem como à contribuição à mobilidade sustentável.

Esse sistema pode partir de uma iniciativa pessoal ou por meio de organização externa. Em outros termos, duas ou mais pessoas podem acordar em dividir o trajeto feito em veículo particular, em um determinado dia e horário, a partir de pontos de partida e chegada predefinidos. Importante destacar que o trajeto pode ser compartilhado em sua totalidade ou parcialmente, com a divisão proporcional dos custos da viagem entre os passageiros.

\footnotetext{
${ }^{5}$ Ver: <http://www.nrw.pendlernetz.de>. 


\subsection{Benefícios de covoiturage no caso da ação por uma grande empresa}

Conforme dados pesquisados em Certu (2009):

Para os utilizadores/empregados:

- Diminuição dos custos de transporte nos trajetos casa-trabalho (divisão de custos);

- Melhora do conforto nos trajetos (estresse, tempo, estacionamento);

- Melhoria das relações sociais.

Para a empresa:

- Diminuir e otimizar os custos ligados ao transporte;

- Facilitar o acesso à empresa;

- Beneficiar-se dos efeitos de imagem da marca;

- Reforçar o espírito de equipe entre os empregados.

Para a coletividade:

- Diminuir os congestionamentos e os efeitos negativos associados a eles;

- Melhora da segurança nas estradas;

- Recuperação dos espaços públicos (estacionamentos);

- Redução da dependência energética.

Uma organização em termos de gestão de ofertas e demanda permite o oferecimento de um serviço de compartilhamento de veículo acessível e organizado. Uma evolução importante nesse sistema acontece atualmente, impulsionado pela utilização de alta tecnologia, com o uso de dados de geolocalização em tempo real, pela generalização dos smartphones e de centrais de tratamento de dados de alta velocidade, que permitem a otimização em tempo real da oferta e demanda e do percurso (THALER; TEREDESAI, 2009).

Isso possibilitou o aparecimento de sistemas de partilhamento de trajeto em veículo dinâmico (Dynamic carpooling), sistema no qual é associada a gestão de localização em tempo real da disponibilidade e da demanda visando à obtenção de uma correspondência instantânea e inteligente entre condutor-veículo disponível e pedestres necessitando de transporte a partir de um ponto A para um ponto B. Toda a gestão é realizada por uma interface disponível tanto em computadores como em tablets e smartphones (LIU et al., 2013), com a garantia de um serviço confiável e responsável.

Assim como o autopartage, o covoiture, na forma como está sendo testada em empresas e centros universitários franceses, constitui uma inovação eco-sustentável à mobilidade urbana em Curitiba. 


\section{O contexto jurídico do covoiture}

A noção de covoiturage foi empregada oficialmente por meio de uma portaria do Ministro dos Transportes, de 18 de julho de 1989, que define o covoiturage como a utilização em comum de um veículo. Essa definição aborda diferentes situações: o partilhamento do veículo familiar entre vizinhos, colegas de trabalho ou até mesmo desconhecidos de forma pontual ou regular.

O uso desse meio de compartilhamento de veículos entre funcionários de empresas e servidores de entidades públicas é incentivado no âmbito dos planos de transporte urbano (Artigo 28.1 da Lei LOTI, derivado da Lei de 30 de dezembro de 1996 sobre "o ar e a utilização racional da energia" e da Lei SRU, de 13 de dezembro de 2000) (MINETT; PEARCE , 2011).

Ademais, o chamado Grenelle de l'Environnement - conjunto de encontros políticos organizados na França, visando à tomada de decisões à longo prazo em matéria ambiental e desenvolvimento sustentável - menciona o encorajamento por parte do Estado ao desenvolvimento do covoiturage e a supressão de obstáculos jurídicos a esse desenvolvimento. É mencionada, ainda, a implantação de vias reservadas em rodovias e avenidas urbanas para o transporte coletivo, covoiturage e táxis.

A definição de covoiturage aponta para a necessidade de um regime jurídico adequado à essa atividade. O compartilhamento de veículo voluntário, v.g. para levar e buscar crianças à escola, não está sujeito a um controle específico, tendo em conta que a atividade desenvolvida pelos particulares não é considerada "transporte coletivo", tampouco "transporte comercial" à luz da lei.

Uma empresa de covoiturage, por sua vez, que instala uma central de reservas ou uma plataforma de relacionamento condutor/passageiro, não organiza um transporte no sentido estrito da lei, mas assegura o relacionamento desses atores. Nessa condição, a empresa se responsabiliza apenas pela prestação desse serviço aos usuários. Por outro lado, a empresa não tem responsabilidade sobre o bom desenrolar do trajeto compartilhado. Isso significa que um acidente no percurso será tratado na esfera de responsabilidade civil, com a reparação de danos.

Como se vê, a implementação desses novos tipos de serviços de mobilidade, seja o autopartage ou o covoiturage, além de um apoio efetivo e formal das autoridades públicas, suscita um amplo questionamento sobre o contexto jurídico adequado para sua implementação.

Por certo, há necessidade de se pensar em políticas públicas voltadas à informação e generalização desses meios de mobilidade. Ao mesmo tempo, é necessária a adequação legislativa em pontos específicos, tais como a remuneração do condutor ou os efeitos legais do seguro referente ao(s) passageiro(s).

Evidentemente, a questão do compartilhamento de veículos entre (des)conhecidos deve necessariamente passar pela definição clara e harmônica de todos os seus aspectos, inclusive o jurídico, de forma a facilitar e encorajar possíveis candidatos ao uso desse modelo de mobilidade.

Questões mais amplas relacionadas à responsabilidade jurídica do condutor, do passageiro, do organizador do serviço e, ainda, dos organismos públicos eventualmente envolvidos é uma questão a ser enfrentada e demandam a evolução do presente trabalho. 


\section{Considerações finais}

Enquanto a construção da estrutura proposta no Plano de Mobilidade Urbana e Transporte Integrado de Curitiba, especificamente em relação ao metrô, demanda custos financeiros, ambientais e políticos exorbitantes, a ponto de o projeto ter sido suspenso pela atual gestão municipal (2012/2016), os chamados "Novos Sistemas de Mobilidade" encorajam o desenvolvimento sustentável, melhorando o meio ambiente urbano e afetando positivamente a saúde e o bem-estar de todos - motoristas e passageiros - em oposição ao deslocamento individual em carros.

Esses novos sistemas de mobilidade também possuem reflexo na esfera econômica, uma vez que representam um mercado novo e, por consequência, a criação de novos empregos.

Cabe destacar que a experiência francesa concluiu que a evolução na utilização de tais sistemas de mobilidade é lenta em razão de fatores como confiabilidade e relutância na aderência aos sistemas de eco-mobilidade ora tratados, o que demanda ações de informação ao público, bem como regulamentação prévia.

Contudo, conclui-se que os novos sistemas de mobilidade ora apresentados têm um custo de implementação mais baixo e representam um ganho indireto imediato à sociedade, tanto em relação ao impacto ecológico (veículos deixam de ser utilizados de forma individual) quanto ao impacto econômico relacionado à infraestrutura pública (uma vez que sua implementação demanda poucas obras), desempenhando, dessa forma, um papel importante na gestão da mobilidade urbana. ${ }^{6}$

\section{REFERÊNCIAS}

Association Lien Plus. Disponível em: <http://www.lien-plus.fr/reglementation-transportcovoiturage.php>. Acesso em: 31 jul. 2013.

BIESZCZAT, Alice; SCHWIETERMAN, Joseph P. Carsharing: review of its public benefits and level of taxation. Transportation Research Record, 2012.

CAHOUR, Beatrice; NGUYEN, Claudine; CRÉNO, Lisa. Methodology for studying the user experience with new mobility systems: the cases of electric cars and dynamic car-pooling use. In: SMART 2013, INTERNATIONAL CONFERENCE ON SMART SYSTEMS, DEVICES AND TECHNOLOGIES, 2., 2013, Italy. Proceedings... Italy, 2013.

CERTU. Le covoiturage en France et en Europe: état des lieux et perspectives. Lyon, France: Centre d'études sur les réseaux, les transports, l'urbanisme et les constructions publiques (CERTU), $2007 . \quad$ Disponível em: <http://urbamet.documentation.equipement.gouv.fr/documents/Urbamet/0272/Urbamet0272827/TU_CE02_10686.pdf>. Acesso em: 31 jul. 2013.

\footnotetext{
${ }^{6}$ Ver o exemplo da França, em: BlaBlaCar. Le site du covoiturage en Europe. Disponível em: <http://www.covoiturage.fr/>. Acesso em: 31 jul. 2013.
} 
CERTU. Le covoiturage dynamique: etude préalable avant expérimentation. Rapports d'étude. Lyon, France: Centre d'études sur les réseaux, les transports, l'urbanisme et les constructions publiques (CERTU), 2009. Disponível em: <http://www.espacedestemps.grandlyon.com/_Services_de_mobilite/doc/CERTU\%20Covoit Dynamique_02-02.pdf>. Acesso em: 31 jul. 2013.

DELOACH, Stephen B.; TIEMANN, Thomas K. Not driving alone? American commuting in the twenty-first century. Transportation, v. 39, Issue 3, p. 521-537, May 2012.

DI FEBBRARO, Angela; SACCO, Nicola; SAEEDNIA, Mahnam. One-Way carsharing: solving the relocation problem. TRB - Transportation Research Record, 2012.

EL FASSI, Ahmed; AWASTHI, Anjali; VIVIANI, Marco. Evaluation of carsharing network's growth strategies through discreteevent simulation. Expert Systems with Applications, v. 39, n. 1, p. 6692-6705, Jan. 2012.

FIRNKORN, Jörg. Triangulation of twomethodsmeasuring the impacts of a freefloatingcarsharing system in Germany. Transportation Research Part A: Policy and Practice, v. 46, n. 10, p. 1654-1672, 2012.

LIU, Nianbo et al. Mobility crowdsourcing: toward zero-effort carpooling on individual smartphone. International Journal of Distributed Sensor Networks, number 615282, 2013.

MARTIN, Elliot William; SHAHEEN, Susan A. Greenhouse gas emission impacts of carsharing in North America. IEEE Transactions on Intelligent Transportation Systems, v. 12, n. 4, p. 1074-1086, Dec. 2011.

MINETT, Paul; PEARCE, John. Estimating the energy consumption ipact of casual carpooling. Energies, v. 4, n. 1, p. 126-139, 2011.

SCHAEFERS, Tobias. Exploring carsharing usage motives: a hierarchicalmeans-end chainanalysis. Transportation Research Part A: Policy and Practice, 2013.

THALER, David; TEREDESAI, Ankur. Maximizing ridesharing matches for dynamic carpooling. INTERNATIONAL CONFERENCE ON INTELLIGENT ENVIRONMENTS, 5., Barcelona, Proceedings... Barcelona: IOS Press, 2009. 